\title{
VV+jets and vector boson scattering at the CMS experiment
}

\section{Riccardo Bellan* on behalf of the CMS collaboration}

Università degli studi di Torino and INFN

E-mail: riccardo.bellanecern.ch

\begin{abstract}
The production of massive vector boson pairs is a key process for the understanding of the nonabelian gauge structure of the standard model and for the comprehension of the electroweak symmetry breaking mechanism. The study of the production of vector boson pairs with the presence of two jets in the event allows measuring the electroweak production of vector bosons in association with jets, in particular made up through vector boson scattering (VBS) processes. In this contribution, we presented the recent results of the production of dibosons in association with two jets at $\sqrt{s}=13 \mathrm{TeV}$.
\end{abstract}

EPS-HEP 2017, European Physical Society conference on High Energy Physics 5-12 July 2017

Venice, Italy

\footnotetext{
* Speaker.
} 
The Standard Model (SM) of particle physics has been successfully tested in all aspects probed so far. Although it has been proven its capability of predicting the production cross section of several processes, spanning over 9 orders of magnitude, and to be a consistent theory, still we have to understand in detail the Electroweak Symmetry Breaking (EWSB) mechanism It is therefore crucial to deeply understand the final states mediated by heavy gauge boson pair production, such that the fundamental question become: do we master them, using our most advanced perturbative computations? To unveil the most intimate part of the SM, we may follow three main paths: measure with high precision the differential cross sections, measure exclusive multi boson production cross section of processes such as vector boson scattering and quartic gauge coupling mediated, and investigate the high mass region of diboson system. In this contribution, I reported the recent results obtained by the CMS collaboration on the measurement of the production cross section of $\mathrm{Z}$ pair bosons in association with jets and search for VBS production [3], and the observation of the electroweak production of two same sign $\mathrm{W}$ in association with two jets [5]. Data have been collected with the CMS detector [1] at a centre-of-mass energy of $13 \mathrm{TeV}$ and correspond to an integrated luminosity of $35.9 \mathrm{fb}^{-1}$.

Both diboson channels have been investigated in the fully leptonic final state, however, the two analyses present opposite set of challenges. While the four charged leptons final state can be fully reconstructed, resulting in a very clean channel, the $\mathrm{ZZ}+\mathrm{jets}$ electroweak production is penalised by a low cross section times branching ratio compared to the $\mathrm{ZZ}+\mathrm{QCD}$-induced jet production. On the contrary, the same sign WW + jets kinematics cannot be fully reconstructed, however, its electroweak production is dominant with respect to the QCD-induced jets one, thus resulting in a favourable signal to background ratio. In order to start the investigation of the vector boson scattering with production of $\mathrm{Z}$ pairs, therefore, it was of paramount importance to study in detail the differential cross section as a function of the hadronic properties of the events [3], such as the jet multiplicity, the jet transverse momentum and pseudorapidity, the $\eta$ separation of the two $p_{T}$ leading jets and the invariant mass of their system, and finally the transverse momentum of the ZZ system [2]. This study opened up the possibility to use a more advanced techniques, based on a multi variate analysis, than a simple cut and count strategy, to discriminate the signal (the electroweak production) from the large background, bringing to a measured electroweak production cross section of $\sigma\left(\mathrm{EWpp} \rightarrow \mathrm{ZZ}+\right.$ jets $\rightarrow 111^{\prime} \mathrm{1}^{\prime}+$ jets $)=0.40_{-0.16}^{+0.21}(\mathrm{syst})_{-0.09}^{+0.13}(\mathrm{syst}) \mathrm{fb}$, with a significance of $2.7 \sigma$ (1.6 $\sigma$ expected). Whereas, for the extraction of the signal in the same sign WW analysis we applied a more aggressive set of cuts in order to suppress the background induced by misidentified leptons and from $t \bar{t}$ production. This analysis resulted in the observation of the electroweak production of two same sign WW in association with jets, with a significance of $5.5 \sigma$ (5.7 $\sigma$ expected) and a measured cross section of $\sigma\left(\mathrm{EWpp} \rightarrow \mathrm{WW}+\right.$ jets $\rightarrow 11^{\prime} v v+$ jets $)=3.83 \pm 0.66$ (stat) \pm 0.35 (syst)fb. Two key variables for the investigation of the vector boson scattering process are presented in fig. 1.

Both analyses have also considerably constrained the size of possible anomalous quartic gauge couplings, given the very good agreement between the observables measured from data and their predictions from the SM. Summary results are presented in fig. 2.

The investigation of the multi-boson final state is only at the beginning and in the next few years it will be one of the main frontier in particle physics. 

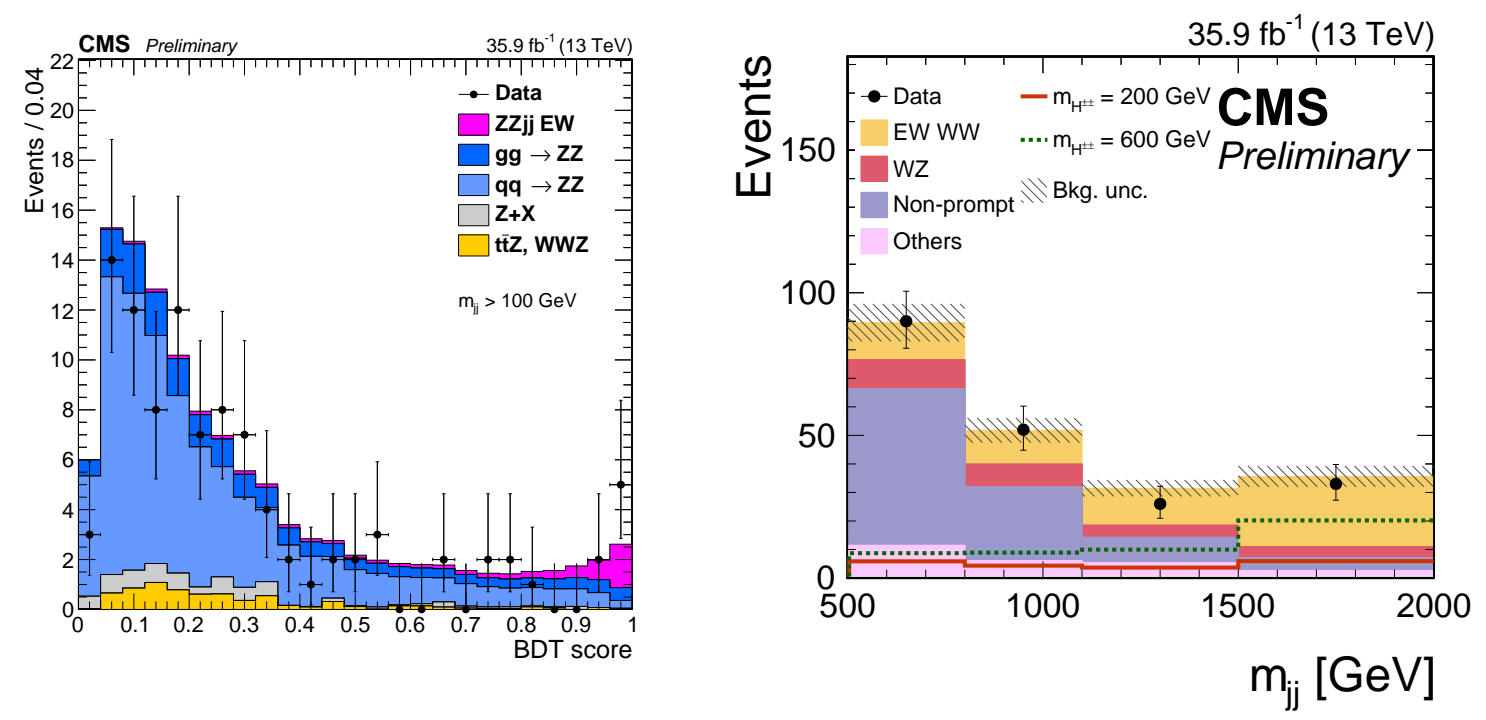

Figure 1: Left: Score of the boosted decision tree (BDT) analysis of the ZZ + jets events [3]. Right: Invariant mass of the dijet system for the selected events in the same sign WW + jets search region [5]. Both plots are the ones presented at the EPS conference, now superseded by [4] and [6], respectively.

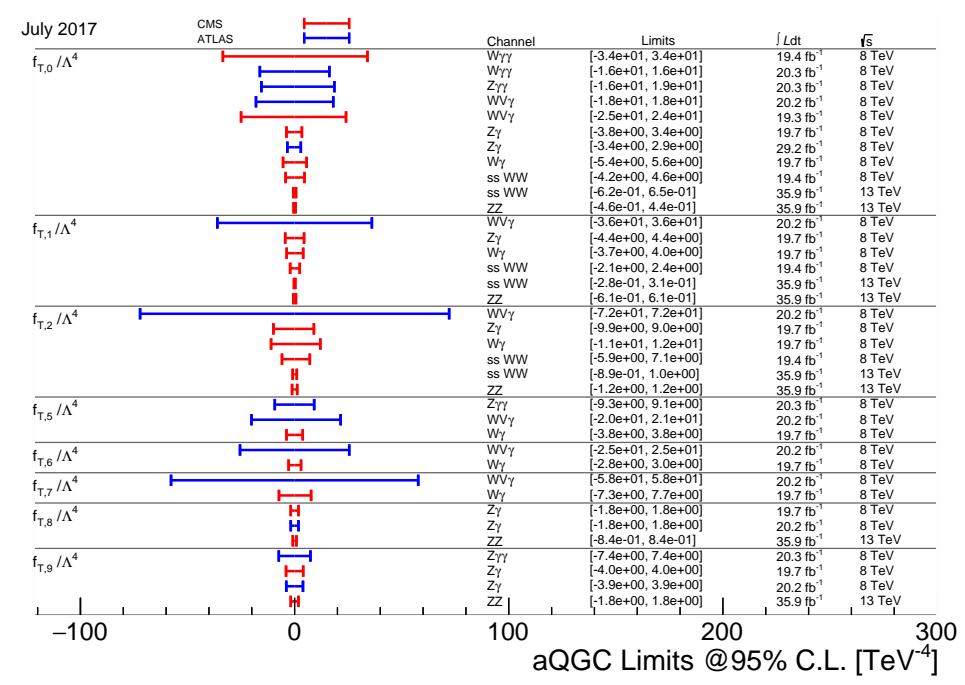

Figure 2: Summary of the investigation of possible anomalies in the quartic gauge couplings in the electroweak sector. 


\section{References}

[1] CMS Collaboration, The CMS experiment at the CERN LHC, JINST 3 S08004 (2008).

[2] CMS Collaboration, Measurement of the $\mathrm{pp} \rightarrow \mathrm{ZZ}$ production cross section, $\mathrm{Z} \rightarrow 41$ branching fraction and constraints on anomalous triple gauge couplings at $\sqrt{s}=13 \mathrm{TeV}$, arXiv:1709.08601, submitted to Eur. Phys. J. C.

[3] CMS Collaboration, Measurements of differential cross sections and search for the electroweak production of two Z bosons in association with jets, CMS PAS SMP-16-019, http://cds.cern.ch/record/2264556.

[4] CMS Collaboration, Measurement of vector boson scattering and constraints on anomalous quartic couplings from events with four leptons and two jets in proton-proton collisions at $\sqrt{s}=13 \mathrm{TeV}$, arXiv:1708.02812, submitted to Phys. Lett. B.

[5] CMS collaboration, Observation of electroweak production of same-sign $\mathrm{W}$ boson pairs in the two jet and two same-sign lepton final state in proton-proton collisions at $13 \mathrm{TeV}$, CMS PAS SMP-17-004, https://cds.cern.ch/record/2264525.

[6] CMS Collaboration, Observation of electroweak production of same-sign $\mathrm{W}$ boson pairs in the two jet and two same-sign lepton final state in proton-proton collisions at $13 \mathrm{TeV}$, arXiv:1709.05822, submitted to Phys. Rev. Lett. 